\title{
INERTIAL AND BORDISM PROPERTIES OF SPHERES
}

\section{ALLAN BRENDER ${ }^{1}$}

Abstract. The $k$-connective bounding group $\theta^{n}(k)$ and the $k$ connective inertial group $I^{n}(k)$ are defined as subgroups of $\theta^{n}$, the group of smooth $n$-spheres, $n \geqq 7$. It is shown $I^{n}(k)$ is contained in $\theta^{n}(k)$. Consequently, the image of the Milnor-Novikov pairing $\tau_{n, k}$ is contained in $\theta^{n+k}(k)$ when $n \geqq k+2$. It follows that $\tau_{7,3}=0$.

1. Introduction. Let $\theta^{n}$ be the group of oriented diffeomorphism classes of manifolds homeomorphic to the usual $n$-sphere, $S^{n}$. Assume $n \geqq 7$. Define $\theta^{n}(k)$ to be the subgroup of $\theta^{n}$ consisting of those $\Sigma^{n}$ which are the boundaries of $k$-connected $(n+1)$-dimensional compact manifolds, $1 \leqq k<[n / 2]$. Thus, $\theta^{n}(k)$ is the kernel of the natural map $i_{k}: \theta^{n} \rightarrow \Omega_{n}(k)$ where $\Omega_{n}(k)$ is the $n$-dimensional group in $k$-connective cobordism theory [10] and $i_{k}$ sends $\Sigma^{n} \in \theta^{n}$ to its cobordism class. Using surgery, we see $\Omega_{*}(1)$ is the usual oriented cobordism group so $\theta^{n}=\theta^{n}(1)$. Similarly, $\Omega_{n}(2) \approx \Omega_{n}^{\text {spin }}$ (recall $\left.n \geqq 7\right)$; since $B$ Spin is, in fact, 3-connected, for $n \geqq 8 \quad \Omega_{n}(2) \approx \Omega_{n}(3)$ and $\theta^{n}(2)$ $=\theta^{n}(3)=b$ Spin $_{n}$. Similarly, $\theta^{n}(k)=\theta^{n}(k+1)$ for $n \geqq 2 k+4$ and $k$ $\equiv 2,4,5,6(\bmod 8) \cdot \theta^{n}$ is filtered

$$
\theta^{n}=\theta^{n}(1) \supset \theta^{n}(2) \supset \cdots \supset \theta^{n}([n / 2]-1) \supset \theta^{n}(\partial \pi) .
$$

The last inclusion is demonstrated in [5].

Let $I^{n}(k)$ be the group of those $\Sigma^{n} \in \theta^{n}$ such that for some $k$-connected closed manifold $M^{n}, M^{n} \# \Sigma^{n}$ is diffeomorphic to $M^{n}$. All diffeomorphisms are assumed to preserve orientation. In the notation of [6], $\Sigma \in I(M)$, the inertia group of $M$. Our main result is

TheOREM. $I^{n}(k)$ is a subgroup of $\theta^{n}(k)$.

We postpone the proof to the next section. Wall [13] has proved this result when $n=2 m, k=m-1$ with a different construction. In general, the groups of the theorem are not equal. This is discussed in $\$ 3$.

Let $\tau_{n, k}: \theta^{n} \otimes \pi_{k}(S O(n-1)) \rightarrow \theta^{n+k}$ be the Milnor-Munkres-Novikov pairing [7, p. 583], [4]. Munkres has shown [7, p. 577], [9] that if

Received by the editors October 17, 1969.

AMS 1969 subject classifications. Primary 5710.

Key words and phrases. $k$-connective cobordism, inertia group, exotic spheres.

1 This research was supported in part by an I.I.T. Faculty Research Fellowship and by NSF contract GP-9614. 
$M^{n+k}$ contains an embedded $S^{k+1}$ with normal bundle having characteristic class $\alpha \in \pi_{k}(S O(n-1))$, then for any $\Sigma^{\prime} \in \theta^{n}, \tau_{n, k}\left(\Sigma^{\prime} \otimes \alpha\right)$ $\in I(M)$. In particular, if $M^{n+k}$ is the total space of the $S^{n-1}$-bundle over $S^{k+1}$ with characteristic class $S \alpha, \alpha \in \pi_{k}(S O(n-2)), \tau_{n, k}\left(\theta^{n} \otimes \alpha\right)$ $\subset I\left(M^{n+k}\right)$. Thus we have

Corollary. If $n \geqq k+2$, image $\tau_{n, k} \subset \theta^{n+k}(k)$.

De Sapio [4] has shown $\tau_{n, k}$ is trivial for $k \geqq n-3$. We shall, therefore, always take $n \geqq k+4$.

2. Proof of the theorem. Let $k \geqq 2$. Let $\Sigma^{n} \in I^{n}(k)$ and assume $d: M \# \Sigma \rightarrow M$ is a diffeomorphism for some $k$-connected closed $M^{n}$. Let $N$ be an elementary cobordism between the disjoint union $M \cup \Sigma$ and $M \# \Sigma ; N$ can be taken to be the connected sum along the boundaries $M \times\{1\}$ and $\Sigma \times\{1\}$ of $M \times I$ and $\Sigma \times I$. Since $N$ has $M \cup \Sigma \cup_{h} D^{1}$ as a deformation retract, it is also $k$-connected. Choose $p$ in $M$ so that $p$ has a small $n$-disc neighborhood $U$ in $M$ with $U \times I$ disjoint from the $(n+1)$-disc removed from $M \times I$ when forming $N$. We can assume $d \mid U \times\{1\}$ is the identity map $d(u, 1)=u$. Identify $x \in M \# \Sigma$ with $(d(x), 0) \in M \times\{0\} \subset N$ and let the resulting manifold be $V^{n+1}$. Then $b V^{n+1}=\Sigma^{n} . p \times I C N$ becomes an embedded circle $C$ in $V$ with trivial normal bundle $C \times U$. It is elementary that $\pi_{1}(V)$ is generated by $j_{*} \pi_{1}\left(S^{1}\right)$ where $j: S^{1} \rightarrow C$ is the embedding. Regarding $V$ as $N \cup M$ $X I$, the Mayer-Vietoris sequence gives

$$
0 \rightarrow H_{1}(V) \rightarrow Z+Z \stackrel{\lambda}{\rightarrow} Z+Z \stackrel{\mu}{\rightarrow} Z \rightarrow 0
$$

where $\lambda(m, n)=(m+n,-m-n)$. Thus $H_{1}(V) \approx \pi_{1}(V) \approx Z$. The class $[j]$ is a generator. Moreover, the same Mayer-Vietoris sequence shows $H_{i}(V)=0,2 \leqq i \leqq k$. Now, perform surgery on $V$ by cutting out int $(C \times U)$ and attaching $D^{2} \times S^{n-1}$ along the new boundary to obtain $W^{n+1}$ which is also bounded by $\Sigma^{n}$. W is clearly simply connected and it is a simple matter to check, using $X=V \times I \cup_{\Phi} D^{2} \times D^{n}$, the trace of the surgery, that $W$ is, in fact, $k$-connected.

3. Remarks. We consider a few special cases and mention several known results concerning the groups $I^{n}(k)$ and $\theta^{n}(k)$.

In a strict sense, the inclusion of the theorem is proper. $I^{2 n-1}(n-1)$ is always trivial since an $(n-1)$-connected closed $M^{2 n-1}$ is a sphere $\Sigma^{2 n-1}$ and therefore has trivial inertia group. On the other hand, $\theta^{2 n-1}(n-1)$ contains $\theta^{2 n-1}(\partial \pi)$ which is, in general, nontrivial and, for even $n$, quite large. 
Wall [13] has shown $I^{2 n}(n-1)=0$ for $n \equiv 2,3,5,6,7(\bmod 8)$ and has order no greater than 4,8 or 2 respectively when $n \equiv 0,1,4$ ( $\bmod 8)$ respectively. Also, $\theta^{2 n}(n-1)=\theta^{2 n}(\partial \pi)=0$ for $n \equiv 5,6$ $(\bmod 8)$. If $n \geqq 4$ and $n \equiv 0,1,4(\bmod 8)$ then $I^{2 n}(n-1)=\theta^{2 n}(n-1)$. For $n>8$, we choose a class $\alpha \in \pi_{n-1}(S O(n-1))$ such that $S S \alpha$ $\in \pi_{n-1}(S O(n+1))$ is a generator and let $M^{2 n}$ be the total space of the $S^{n}$-bundle over $S^{n}$ with characteristic class $S \alpha$. Then $I\left(M^{2 n}\right)=\theta^{2 n}$ $(n-1)$ [13, Theorem 10]. If $n=4$ or 8 take for $M^{2 n}$ Tamura's manifolds [12] $\bar{B}_{7,1}^{8} \cup_{i} D^{8}$ or $\bar{B}_{127,1}^{16} \cup_{i} D^{16}$; again, $I\left(M^{2 n}\right)=\theta^{2 n}(n-1)$.

We now consider several low-dimensional cases. It is well known [5] that $\theta^{7}=\theta^{7}(3)=\theta^{7}(\partial \pi) \approx Z_{28}$. Tamura [11] has shown $I^{7}(2)=\theta^{7}$. Now, $\theta^{8} \approx Z_{2}$. The sole exotic 8 -sphere is a spin-boundary [1] so $\theta^{8}=\theta^{8}(3)$. As remarked above, $I^{8}(3)=\theta^{8}(3)$. $\theta^{8}$ has order eight. It follows from [1], [3], [8] that $\theta^{9} \approx Z_{2}+Z_{2}+Z_{2}$. Take as generators of the summands $\Sigma_{1}, \Sigma_{2}, \Sigma_{3}$. Then $\Sigma_{1}$ is not a spin-boundary while $\Sigma_{2}$ and $\Sigma_{3}$ are, so $\theta^{9}(3) \approx Z_{2}+Z_{2}$. $\Sigma_{3}$ may be taken to be the generator of $\theta^{9}(\partial \pi) \approx Z_{2}$. From [2], $\Sigma_{3} \in I^{9}(3)$. I do not know if $\Sigma_{2} \in I^{9}(3) . \theta^{10} \approx Z_{2}$ $+Z_{3}$ with generators $\Sigma_{2}$ and $\Sigma_{3} . \Sigma_{2}$ is not a spin-boundary [8] while $\Sigma_{3}$ is, so $\theta^{10}(3) \approx Z_{3}$. $\theta^{10}(4)=\theta^{10}(\partial \pi)=0$. From the corollary, image $\tau_{7,3} \subset \theta^{10}(3)$, so $\tau_{7,3}: Z_{28} \rightarrow Z_{3}$ is trivial.

The author has learned that $A$. Winkelnkemper has shown that any exotic sphere $\Sigma^{n} \in \theta^{n}$ is in the inertia group of some closed $M^{n}$, but the author does not know whether Winkelnkemper's proof yields any information on the connectivity of $M$.

\section{REFERENCES}

1. D. W. Anderson, E. H. Brown, Jr. and F. P. Peterson, The structure of the Spin cobordism ring, Ann. of Math. (2) 86 (1967), 271-298. MR 36 \#2160.

2. E. H. Brown, Jr. and B. Steer, A note on Stiefel manifolds, Amer. J. Math. 87 (1965), 215-217. MR 30 \#5322.

3. G. Brumfiel, On the homotopy groups of BPL and PL/O. II, Topology 8 (1969), 305-311.

4. R. De Sapio, Differential structures on a product of spheres. II, Ann. of Math. (2) 89 (1969), 305-313. MR 39 \#7611.

5. M. A. Kervaire and J. W. Milnor, Groups of homotopy spheres. I, Ann. of Math. (2) 77 (1963), 504-537. MR $26 \# 5584$.

6. A. Kosinski, On the inertia group of $\pi$-manifolds, Amer. J. Math. 89 (1967), 227-248. MR 35 \# 4936.

7. R. Lashof (Editor), Problems in differential and algebraic topology, Seattle Conference, 1963, Ann. of Math. (2) 81 (1965), 565-591. MR 32 \#443.

8. J. W. Milnor, Remarks concerning spin manifolds, Differential and Combinatorial Topology (A Symposium in Honor of Marston Morse), Princeton Univ. Press, Princeton, N. J., 1965. MR 31 \#5208. 
9. J. Munkres, Concordance inertia groups, Advances in Math. 4 (1970), 224-235. 10. R. E. Stong, Notes on cobordism theory, Princeton Univ. Press, Princeton, N. J., 1968.

11. I. Tamura, Sur les sommes connexes de certaines variêtes differentiables, C. R. Acad. Sci. Paris 255 (1962), 3104-3106. MR 26 \#781.

12. - 8-manifolds admitting no differentiable structure, J. Math. Soc. Japan 13 (1961), 377-382. MR $26 \# 780$.

13. C. T. C. Wall, Classification problems in differential topology. VI. Classification of (s-1)-connected (2s+1)-manifolds, Topology 6 (1967), 273-296. MR 35 \#343.

Illinois Institute of Technology, Chicago, Illinois 60616 